\title{
Diversidade de ixodida em roedores e marsupiais capturados no Parque Estadual da Pedra Branca, Rio de Janeiro, Brasil
}

[Diversity of ixodida in small rodents and marsupials in the Pedra Branca state park, state of Rio de Janeiro, Brazil]

\author{
H.H. Oliveira ${ }^{1}$, V. Gomes ${ }^{2}$, M. Amorim ${ }^{2}$, G.S. Gazêta ${ }^{2}$, N.M. Serra-Freire ${ }^{2,6}$, I.P.F. Quinelato ${ }^{2}$, \\ V.F. Morelli-Amaral ${ }^{3}$, A.B. Almeida ${ }^{4}$, R.W. Carvalho ${ }^{4}$, A.G. Carvalho ${ }^{5}$ \\ ${ }^{1}$ Pós-doutoranda - Instituto Oswaldo Cruz - Fiocruz - Manguinhos, RJ \\ ${ }^{2}$ Instituto Oswaldo Cruz - Fiocruz - Manguinhos, RJ \\ ${ }^{3}$ Instituto de Tecnologia em Fármacos - Fiocruz - Manguinhos, RJ \\ ${ }^{4}$ Escola Nacional de Saúde Pública - Fiocruz - Manguinhos, RJ \\ ${ }^{5}$ Universidade Federal Rural do Rio de Janeiro - Seropédica, RJ \\ ${ }^{6}$ Bolsista de Produtividade Científica do CNPq
}

\begin{abstract}
RESUMO
Durante 24 meses foram capturados, inspecionados e liberados no mesmo espaço do Parque Estadual da Pedra Branca, Rio de Janeiro, 96 marsupiais e 64 roedores. Neles foram recolhidos manualmente 105 carrapatos, de 10 espécies em duas famílias. A espécie de carrapato dominante entre os roedores foi Amblyomma longirostre e entre os marsupiais foi Ixodes loricatus. Houve correlação direta significativa entre a temperatura e a intensidade de parasitismo por carrapatos.
\end{abstract}

Palavras-chave: Ixodidae, Didelphimorphia, Rodentia, Mata Atlântica

\begin{abstract}
During 24 months 96 marsupials and 64 rodents were captured, inspected and set free in the same space of the Pedra Branca State Park, Rio de Janeiro. From them, 105 ticks from 10 species in two families were manually collected. The dominant tick species on the rodents was Amblyomma longirostre and on the marsupials was Ixodes loricatus. There was a significant correlation of the temperature in relation to intensity of parasitism for ticks, demonstrating that the higher the temperature, greater is the number of ticks.
\end{abstract}

Keywords: Ixodidae, Didelphimorphia, Rodentia, Atlantic Forest

\section{INTRODUÇÃO}

No Brasil, são reconhecidas duas famílias de carrapatos: Argasidae e Ixodidae, nas quais todos os adultos de todas as espécies são metabolicamente dependentes de fluidos orgânicos de vertebrados para realizarem a reprodução. Estão amplamente dispersos no Brasil, com envolvimento direto como parasito do homem ou de animais domésticos e silvestres, animais de produção zootécnica, de guarda e/ou companhia além do envolvimento como vetor de bioagentes de doenças para seus hospedeiros (Serra-Freire e Mello, 2006).

Recebido em 17 de fevereiro de 2013

Aceito em 16 de dezembro de 2013

E-mail: heloizamorelli@hotmail.com
O gênero Ixodes Latreille, 1795, compreende 234 espécies no mundo, distribuídas em 14 subgêneros. Na fase adulta, algumas espécies parasitam, preferencialmente, mamíferos, como pacas, gambás, cuícas, cotias, enquanto as fases imaturas preferem pequenos mamíferos, principalmente roedores (Guimarães et al., 2001).

O gênero Amblyomma é constituído por 106 espécies, das quais 45 estão restritas à região neotropical. Nesse gênero estão os carrapatos maiores e mais ornamentados, que parasitam anfíbios, répteis, aves e mamíferos, em todas as regiões geográficas do Brasil (Guimarães et al., 2001; Guglielmone et al., 2003b). 
O Parque Estadual da Pedra Branca (PEPB) é um importante ponto de referência na preservação dos vestígios de Mata Atlântica no município do Rio de Janeiro e é onde se encontra o ponto mais alto do município, com 1024m acima do nível do mar. Abriga exuberante floresta e preserva espécies nativas da Mata Atlântica, bem como espécimes da fauna brasileira (Cintra et al. 2007). Além disso, o contínuo da Mata Atlântica brasileira é considerado um dos ecossistemas com maior biodiversidade e taxa de endemismo do planeta (Fonseca, 1985). Para grande parte dos grupos animais, existe notável variação na diversidade local entre áreas ao longo deste contínuo. Assim, tendo em vista a carência de informações científicas sobre as espécies de Ixodidae que utilizam marsupiais e roedores como hospedeiros no Parque Estadual da Pedra Branca (PEPB) e considerando que parasitos e hospedeiros evoluem paralelamente, o estudo das relações entre os pequenos mamíferos $\mathrm{e}$ ectoparasitos pode facilitar a compreensão ecológica, evolutiva e taxonômica de ambos.

Este trabalho objetivou identificar a fauna ixodológica de roedores e marsupiais capturados no PEPB e verificar se há correlação entre mesoclima ou microclima com a relação parasitária.

\section{MATERIAL E MÉTODOS}

O trabalho foi desenvolvido no Parque Estadual da Pedra Branca, em área caracterizada como espaço geopolítico da localidade Pau da Fome, em Jacarepaguá, Rio de Janeiro, com $22^{\circ} 56^{\prime} 26^{\prime \prime}$ de latitude sul e $43^{\circ} 26^{\prime} 28^{\prime \prime}$ de longitude oeste.

A área selecionada foi dividida em seis subáreas nas cotas de $150 \mathrm{~m}, 200 \mathrm{~m}, 300 \mathrm{~m}, 400 \mathrm{~m}, 500 \mathrm{~m}$ e $600 \mathrm{~m}$ de altitude. Roedores e marsupiais foram capturados, no período de outubro de 2005 a outubro de 2007, usando-se como armadilhas modelos Sherman, Tomahowk e Francesinha, com dimensões variadas. Nos 24 meses, as capturas eram feitas durante quatro noites consecutivas, totalizando um esforço de captura de 10.560 armadilhas/dia. As armadilhas iscadas distavam 10 metros uma da outra e foram distribuídas em cinco transectos, sendo estudada uma cota altimétrica em cada mês. Banana, mandioca, milho em sabugo e pasta de amendoim foram utilizados como isca. Também foram instaladas cinco armadilhas Tomahowks nas linhas um, três e cinco de cada cota, iscadas com carne moída misturada com bacon e fixadas em um suporte de madeira que era suspenso em árvore para captura de marsupiais de hábitos arborícolas.

Após a captura, os mamíferos eram anestesiados, marcados na orelha, manuseados na remoção de ectoparasitos, e liberados no mesmo local de captura. Este trabalho foi desenvolvido com a autorização do Instituto Estadual de Florestas do Rio de Janeiro (IEF/RJ/PR n ${ }^{\circ}$ 015/05) e do Instituto Brasileiro do Meio Ambiente e dos Recursos Naturais Renováveis (Ibama) $\left(n^{\circ}\right.$ da licença 058/06-RJ)

Os carrapatos foram removidos manualmente, com auxílio de uma pinça de dissecação, sendo acondicionados em frascos plásticos identificados, contendo etanol $70^{\circ} \mathrm{GL}$ como líquido preservador. Para a identificação, os carrapatos foram analisados por estereomicroscopia, e foi utilizada a chave de identificação proposta por Aragão e Fonseca (1961), Keirans (1992), Guimarães et al. (2001) e Serra-Freire e Mello (2006). Para a preparação e montagem das larvas, entre lâmina e lamínula de forma permanente, foi adotada a técnica de Amorim e Serra-Freire (1995), e para a identificação foi utilizada a chave dicotômica de Amorim e Serra-Freire (1999).

Os dados foram analisados por meio do programa Bioestat 5.0, correlação de Sperman (rs) para comparar a carga parasitária com as temperaturas ambiente, e por qui-quadrado entre as intensidades de parasitismo e as cotas de altitude. Além disso, inferiram-se os índices ecológicos de dominância (coeficiente de dominância $=\mathrm{CD})$, intensidade média parasitária (IMP) e abundância (índice de abundância = IA). Foi arbitrado o nível de significância em 5\% para a tomada de decisão dos resultados estatísticos.

\section{RESULTADOS E DISCUSSÃO}

Foram capturados 160 pequenos mamíferos, sendo 96 marsupiais e 64 roedores, ambos com seis espécies. A espécie de hospedeiro dominante foi Didelphis aurita (Wied-Neuwied, 1826) $(\mathrm{CD}=35 \%)$, seguida de Guerlinguetus ingrami (Thomas, 1901) (CD= 27,5\%), Micoureus paraguayanus (Tate, 1931) (CD=11,3\%), Marmosops incanus (Lund, 1840) $(\mathrm{CD}=10,6 \%)$, Rattus norvergicus (Berkenhout, 1769) $(\mathrm{CD}=$ 
5,0\%), Akodon cursor (Winge, 1887) (CD= $3,8 \%$ ), Oligoryzomys nigripes (Olfers, 1818) $(\mathrm{CD}=2,5 \%)$, Monodelphis americana (Muller, 1776) $(\mathrm{CD}=1,9 \%)$ e Metachirus nudicaudatus (Geoffroy, 1803), Philander frenatus (Olfers, 1818), Rattus rattus (Linnaeus, 1758) e Sphigurus villosus (F. Cuvier, 1823) $(\mathrm{CD}=$ 0,63\%). Entre os carrapatos encontrados, o gênero Amblyomma foi o dominante, só sendo encontrado em $G$. ingrami, entre os roedores, e em D. aurita, entre os marsupiais (Tab. 1), nesses com intensidade média de parasitismo de 4,57 carrapatos/hospedeiro. A espécie de Ixodidae mais frequente em $G$. ingrami foi $A$. longirostre $(9,2 \%)$, e nos marsupiais foi $I$. loricatus $(10,1 \%)$.

Tabela 1. Coeficiente de dominância (CD), e frequência das espécies de carrapatos parasitos de roedores e marsupiais capturados na localidade Pau da Fome, Jacarepaguá, Parque Estadual da Pedra Branca, Rio de Janeiro, RJ, em estudo ecológico sobre a diversidade de carrapatos nesses hospedeiros, no período de outubro de 2005 a outubro de 2007

\begin{tabular}{|c|c|c|c|c|c|c|c|c|}
\hline \multirow{3}{*}{$\begin{array}{l}\text { Espécies } \\
\text { de } \\
\text { Ixodidae }\end{array}$} & \multicolumn{4}{|c|}{ Didelphimorphia } & \multirow{2}{*}{\multicolumn{2}{|c|}{$\begin{array}{c}\text { Rodentia } \\
\begin{array}{c}\text { Guerlinguetus } \\
\text { ingrami }\end{array}\end{array}$}} & \multirow{2}{*}{\multicolumn{2}{|c|}{ Soma }} \\
\hline & \multicolumn{2}{|c|}{$\begin{array}{c}\text { Didelphis } \\
\text { aurita }\end{array}$} & \multicolumn{2}{|c|}{$\begin{array}{l}\text { Philander } \\
\text { frenatus }\end{array}$} & & & & \\
\hline & $\mathrm{n}^{\mathrm{O}}$ & $\%$ & $\mathrm{n}^{\mathrm{o}}$ & $\%$ & $\mathrm{n}^{\mathrm{O}}$ & $\%$ & $\mathrm{n}^{\mathrm{o}}$ & $\mathrm{CD}(\%)$ \\
\hline Amblyomma geayi & 01 & 3,6 & 00 & 0,0 & 03 & 4,2 & 4 & 3,81 \\
\hline Amblyomma longirostre & 00 & 00 & 00 & 0,0 & 12 & 16,7 & 12 & 11,43 \\
\hline Amblyomma scutatum & 01 & 3,6 & 00 & 0,0 & 00 & 0,0 & 1 & 0,95 \\
\hline Amblyomma spp. & 00 & 0,0 & 00 & 0,0 & 50 & 69,4 & 50 & 47,62 \\
\hline Ixodes amarali & 07 & 25,0 & 00 & 0,0 & 00 & 0,0 & 7 & 6,67 \\
\hline Ixodes. auritulus & 01 & 3,6 & 00 & 0,0 & 00 & 0,0 & 1 & 0,95 \\
\hline Ixodes didelphidis & 01 & 3,6 & 00 & 0,0 & 00 & 0,0 & 1 & 0,95 \\
\hline Ixodes loricatus & 14 & 50,0 & 00 & 0,0 & 00 & 0,0 & 14 & 13,33 \\
\hline Ixodes luciae & 03 & 10,7 & 05 & 100 & 00 & 0,0 & 8 & 7,62 \\
\hline Ixodes spp. & 00 & 0,0 & 00 & 0,0 & 07 & 9,7 & 7 & 6,67 \\
\hline Soma & 28 & 100 & 05 & 100 & 72 & 100 & 105 & 100 \\
\hline
\end{tabular}

Houve correlação direta e significativa ( $\mathrm{rs}=$ $0,772, p=0,0101$ ) entre a temperatura de meso e microclima em relação ao parasitismo por carrapato, o que demonstra que, quanto maior a temperatura, maior é o número de carrapatos $(\mathrm{P}<0,05)$.

$\mathrm{O}$ teste de qui-quadrado para a hipótese $\left(\mathrm{H}_{0}\right)$ de que a quantidade de carrapatos nos hospedeiros não sofria influência da altitude da cota em que os hospedeiros foram capturados indicou a rejeição de $\mathrm{H}_{0}$. Hospedeiros capturados na cota $200 \mathrm{~m}$ estavam significativamente mais parasitados daqueles das outras cotas (Tab. 2). Quando se consideraram somente três faixas altitudinais, reunindo dados das cotas estudadas duas a duas, mais da metade dos carrapatos foi recolhido de hospedeiros nas duas cotas mais baixas, possivelmente indicando que isso acontece porque são áreas mais antropizadas.

Em seu trabalho sobre Ixodidas brasileiros, Aragão (1936) refere o parasitismo de marsupiais por Ixodes loricatus Neumann, 1899, e Amblyomma striatum Koch, 1844 , e assinala $D$. aurita como hospedeiro nos estados do Rio de Janeiro, Minas Gerais, Pernambuco, São Paulo, Bahia, Sergipe, Piauí e Santa Catarina. No presente trabalho, foi observado I. loricatus em $D$. aurita e $P$. frenatus, ampliando o número de hospedeiros para a espécie.

No estado do Paraná, Barros e Baggio (1992) assinalaram $I$. loricatus em $D$. aurita, $D$. albiventris, Didelphis spp., $P$. frenatus e Lutreolina crassicaudata (Desmarest, 1804), assim como Amblyomma spp., em D. albiventris. Os resultados obtidos no PEPB comprovam parcialmente esta afirmação, mas algumas espécies de hospedeiros não foram capturadas desta vez no parque. Coutinho et al. (1999) encontraram I. amarali em quatro dos $49 \mathrm{D}$. albiventris capturados na Estação Ecológica da UFMG; no PEPB, entretanto, foi encontrado só em D. aurita. Para o mesmo espaço geopolítico do Rio de Janeiro, mas na área de Mata Atlântica, na Ilha Grande, Bittencourt e Rocha (2003) tiveram resultado idêntico ao achado no PEPB. 
Tabela 2. Intensidade média de parasitismo (IMP), frequência absoluta e relativa das espécies de carrapatos parasitos de roedores e marsupiais capturados na localidade Pau da Fome, Jacarepaguá, Parque Estadual da Pedra Branca, Rio de Janeiro, RJ, considerando as cotas de altitude em estudo ecológico sobre a diversidade nesses hospedeiros, no período de outubro de 2005 a outubro de 2007

\begin{tabular}{|c|c|c|c|c|c|c|c|c|}
\hline \multirow{3}{*}{$\begin{array}{c}\text { Espécies } \\
\text { de } \\
\text { Ixodidae }\end{array}$} & \multicolumn{8}{|c|}{ Cota de altitude (metros) } \\
\hline & \multirow{2}{*}{$\begin{array}{c}150 \\
\mathrm{n}^{\mathrm{o}}\end{array}$} & \multirow{2}{*}{$\begin{array}{c}200 \\
\mathrm{n}^{\mathrm{o}}\end{array}$} & \multirow{2}{*}{$\begin{array}{c}300 \\
\mathrm{n}^{\mathrm{o}}\end{array}$} & \multirow{2}{*}{$\begin{array}{c}400 \\
n^{0}\end{array}$} & \multirow{2}{*}{$\begin{array}{c}500 \\
\mathrm{n}^{\mathrm{o}}\end{array}$} & \multirow{2}{*}{$\begin{array}{c}600 \\
\mathrm{n}^{\mathrm{o}}\end{array}$} & \multicolumn{2}{|c|}{ Soma } \\
\hline & & & & & & & $\mathrm{n}^{\mathrm{o}}$ & $(\%)$ \\
\hline Amblyomma geayi & 00 & 00 & 00 & 00 & 00 & 04 & 4 & 3,81 \\
\hline Amblyomma longirostre & 00 & 00 & 00 & 04 & 00 & 08 & 12 & 11,43 \\
\hline Amblyomma scutatum & 00 & 00 & 00 & 00 & 00 & 01 & 1 & 0,95 \\
\hline Amblyomma spp. & 08 & 40 & 00 & 00 & 00 & 02 & 50 & 47,62 \\
\hline Ixodes amarali & 02 & 02 & 00 & 01 & 00 & 02 & 7 & 6,67 \\
\hline Ixodes. auritulus & 00 & 00 & 00 & 01 & 00 & 00 & 1 & 0,95 \\
\hline Ixodes didelphidis & 00 & 01 & 00 & 00 & 00 & 00 & 1 & 0,95 \\
\hline Ixodes loricatus & 03 & 00 & 00 & 04 & 00 & 07 & 14 & 13,33 \\
\hline Ixodes luciae & 03 & 05 & 00 & 00 & 00 & 00 & 8 & 7,62 \\
\hline Ixodes spp. & 03 & 00 & 00 & 04 & 00 & 00 & 7 & 6,67 \\
\hline Total número & $19 \mathrm{~b}$ & $48 \mathrm{a}$ & 00 & $14 \mathrm{c}$ & 00 & $24 \mathrm{~b}$ & 105 & 100 \\
\hline porcentagem & 18,10 & 45,71 & 0,00 & 13,33 & 0,00 & 22,86 & 100 & \\
\hline $\operatorname{IMP}\left(n^{\circ}\right)$ & $3,2 \mathrm{~b}$ & $12,0 \mathrm{a}$ & 0,0 & $2,8 \mathrm{~b}$ & 0,0 & $3,0 \mathrm{~b}$ & & 4,6 \\
\hline
\end{tabular}

Expoentes com letras minúsculas iguais na mesma linha indicam diferença não significativa $(\mathrm{P}>0,05)$, e com letras diferentes indicam diferença significativa $(\mathrm{P}<0,05)$.

Barros-Battesti et al. (2000), ao trabalharem em fragmento de Mata Atlântica em Itapevi, São Paulo, demonstraram que os roedores silvestres apresentavam somente formas jovens de Ixodes spp., com um grande número sendo registrado na estação seca, enquanto os marsupiais ficam infestados por adultos e jovens, na estação chuvosa; o maior índice ocorreu no mês de janeiro, e essas espécies de carrapato não foram encontradas nos meses de junho e outubro. Bossi et al. (2002) relataram a ocorrência de Ixodes spp., em Oryzomys russatus (Wagner, 1848) e Proechimys iheringi $[=$ Trinomys iheringi (Thomas, 1911)], e I. loricatus, em D. aurita, $M$. nudicaudatus e $P$. frenata (= Philander frenatus), na Estação Ecológica da Jureia-Itatins, São Paulo. Como também foi encontrada correlação positiva entre dados climáticos e o ectoparasitismo por artrópodes, os autores relataram que a relação entre a intensidade de parasitismo e o período do ano (seco e úmido) foi significativa somente para $O$. russatus, que apresentou valores mais altos no período seco. $\mathrm{Na}$ presente investigação não se encontraram tantos roedores parasitados, mas pode ser considerada identidade da correlação positiva entre temperatura e número de carrapatos, mesmo que os hospedeiros sejam de espécies diferentes (Quadro 1). No sul do Brasil, município de Pelotas, Rio Grande do Sul, e adjacências, Muller et al. (2005) constataram a presença de $I$. loricatus em $D$. albiventris, espécie de carrapato com maior frequência, e encontraram dois hospedeiros parasitados por jovens e adultos, estando os demais somente com estádios adultos, o que demonstra ser possível encontrar larvas e ninfas de Ixodes sp. em gambás. Diante disso, os achados de carrapatos em gambás e roedores devem ser abordados com clareza de pensamento, posto que I. loricatus tem sido considerado altamente específico para marsupiais do Novo Mundo. Entretanto, o frequente encontro de formas imaturas em roedores sugere que estes vertebrados possam ter um papel relevante no ciclo de doenças com agentes transmitidos por carrapatos e que a preferência de hospedeiro está principalmente relacionada a fatores como o tipo de habitat e a densidade de hospedeiro (Beldomenico et al., 2005).

Embora Ixodes auritulus Neumann, 1904, seja um ectoparasita comum em aves, incluindo as marinhas, também tem sido observado em mamíferos (Arzua et al., 1994), como agora no PEPB (Quadro 1). Evans et al. (2000) relataram que esta relação é para as Américas Central e do Sul. Gonzalez-Acuña et al. (2005) observaram que I. auritulus ocorre da Guatemala até o sul da Argentina e é sempre encontrado em aves 
das ordens Ciconiiformes, Columbiformes, Falconiformes, Galliformes, Passeriformes, Piciformes, Procellariiformes e Tinamiformes, sendo os passeriformes, provavelmente, os principais hospedeiros.

Fonseca (1957/58) registrou no Brasil a presença de I. amarali, em roedores dos gêneros Oryzomys, Bolomys Thomas, 1916, Holochilus Brandt, 1835, Akodon e Rhipidomys Waterhouse, 1837, nos estados de Alagoas, Ceará e Pernambuco. Também em marsupiais da espécie Monodelphis domestica (Wagner, 1842), proveniente dos estados de Pernambuco e Paraíba. As formas jovens foram encontradas quase que exclusivamente em roedores, e quanto às adultas, somente fêmeas parasitavam os marsupiais. Entretanto, Linardi et al. (1984), em Minas Gerais, verificaram parasitismo por quatro fêmeas e cinco ninfas de $I$. amarali, em Oryzomys subflavus (Wagner, 1842) e Zigodontomys lasiurus (= Necromys lasiurus (Lund, 1841)), este último assinalado pela primeira vez como hospedeiro deste ixodídeo. No Rio de Janeiro, o primeiro registro de $I$. amarali foi de Guitton et al. (1986), pelo encontro de três exemplares em Proechimys dimidiados [= Trinomys dimidiados (Günther, 1877)] parasitados, porém sem referência ao estádio de vida do ixodídeo. Registra-se $D$. aurita como novo hospedeiro para I. amarali, e o Rio de Janeiro como novo local de ocorrência deste carrapato.

Barros-Battesti e Knysak (1999) informaram a existência de hospedeiros e a ocorrência de algumas espécies do gênero Ixodes spp. no Brasil, integrantes do acervo da Coleção do Instituto Butantan. Os autores afirmam que, entre as espécies do gênero Ixodes, que acontecem em São Paulo, I. loricatus e Ixodes didelphidis Fonseca e Aragão, 1952, são as mais comuns entre os marsupiais. Esta última espécie foi encontrada no PEPB, mas com coeficiente de dominância muito baixo (Tab. 1).

Autino et al. (2006) registraram a presença de Ixodes luciae Sénevet, 1945, no noroeste argentino em Calomys callosus (Rengger, 1830), Thylamys cinderella Thomas e Thylamys venustus (= Thylamys elegans (Waterhouse, 1839)) e relataram que esta espécie de carrapato tem ampla distribuição, ocorrendo da Argentina até o sul do México, sendo comumente encontrada em marsupiais. Esta afirmação pode ser aceita porque a espécie só foi encontrada em D. aurita, mas a literatura não discute a distribuição quanto à altitude, como foi desta vez para o PEPB (Tab. 2).

Muller et al. (2005) relataram o encontro de ninfa de Amblyomma spp. parasitando $D$. marsupialis no Rio Grande do Sul. O parasitismo por estádios jovens, larvas e ninfas de Amblyomma spp. ocorre com alta frequência em aves da ordem Passeriformes e em mamíferos. Os estádios jovens foram citados nas ordens Carnivora: família Canidae; Rodentia: família Ctenomydae, Echimydae; Xenarthra: família Bradypodidae. Segundo Guglielmone et al. (2003a), isso ocorre com baixa frequência, no entanto, no PEPB, foi constatada alta frequência de larvas deste gênero em $G$. ingrami, o que demonstra uma alteração do comportamento.

Venzal et al. (2003) fizeram o primeiro registro de Amblyomma longirostre (Koch, 1844) no Uruguai, onde eles observaram a ocorrência de ninfas em uma espécie de ave, e formas adultas foram encontradas em Sphigurus spinosus (Rodentia: Erethizonthidae). De acordo com esses autores, A. longirostre é uma espécie de carrapato cujas formas adultas são encontradas principalmente em roedores da família Erethizontidae, mas ocasionalmente, tem sido encontrada em cebídeos e no homem. As ninfas se alimentam em quirópteros e em Sciurus spp. (Rodentia: Sciuridae). Brum et al. (2003) identificaram os carrapatos que parasitavam mamíferos silvestres trazidos para a recuperação, no Núcleo de Reabilitação da Fauna Silvestre em Pelotas, Rio Grande do Sul, e observaram a ocorrência de A. longirostre em Coendu villosus (= Sphigurus villosus). Os estádios adultos de $A$. longirostre e A. geayi são comumente encontrados em roedores do gênero Sphigurus spp. O exemplar de $S$. villosus capturado no PEPB não estava parasitado por A. longirostre, que foi encontrada em $G$. ingrami (Tab. 1). O significado desse encontro somente poderá ser analisado com estudos adicionais.

Segundo Evans et al. (2000), Amblyomma scutatum Neumann, 1899, é uma espécie de carrapato neotropical que ocorre em lagartos e serpentes e, ocasionalmente, em aves e gambás, o que corrobora o presente achado. No PEPB, esta espécie só foi encontrada na cota $600 \mathrm{~m}$ 
(Tab. 2), em um D. aurita, mas tanto esta espécie de hospedeiro como a outra mais parasitada foram capturadas em diferentes níveis de altitude (Tab. 3), indicando que os carrapatos podem coexistir em todas as altitudes trabalhadas e que, se havia maior parasitismo nas cotas mais baixas, era por fatores intervenientes, como a antropização do ambiente.

Tabela 3. Frequência absoluta e relativa das espécies de roedores e marsupiais capturados na localidade Pau da Fome, Jacarepaguá, Parque Estadual da Pedra Branca, Rio de Janeiro, RJ, considerando as cotas de altitude em estudo ecológico sobre a diversidade de carrapatos nesses hospedeiros, no período de outubro de 2005 a outubro de 2007.

\begin{tabular}{|c|c|c|c|c|c|c|c|c|c|}
\hline \multirow{3}{*}{\multicolumn{2}{|c|}{$\begin{array}{c}\text { Espécies } \\
\text { de } \\
\text { pequeno mamífero }\end{array}$}} & \multicolumn{8}{|c|}{ Cota de altitude (metros) } \\
\hline & & \multirow{2}{*}{$\frac{150}{\mathrm{n}^{\mathrm{o}}(\mathrm{p})}$} & \multirow{2}{*}{$\frac{200}{\mathrm{n}^{\mathrm{o}}(\mathrm{p})}$} & \multirow{2}{*}{$\frac{300}{\mathrm{n}^{\circ}(\mathrm{p})}$} & \multirow{2}{*}{$\frac{400}{\mathrm{n}^{\mathrm{o}}(\mathrm{p})}$} & \multirow{2}{*}{$\frac{500}{\mathrm{n}^{\mathrm{o}}(\mathrm{p})}$} & \multirow{2}{*}{$\begin{array}{c}600 \\
\mathrm{n}^{\mathrm{o}}(\mathrm{p})\end{array}$} & \multicolumn{2}{|c|}{ S o m a } \\
\hline & & & & & & & & $\mathrm{n}^{\mathrm{o}}(\mathrm{p})$ & $(\%)(\mathrm{pi})$ \\
\hline \multirow{6}{*}{ 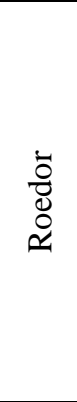 } & Akodon cursor & 01 & 01 & 00 & 00 & 04 & 00 & 06 & 3,75 \\
\hline & $\begin{array}{l}\text { Oligoryzomys } \\
\text { nigripes }\end{array}$ & 00 & 02 & 00 & 01 & 00 & 01 & 04 & 2,50 \\
\hline & Rattus & 01 & 03 & 00 & 01 & 03 & 00 & 08 & 5,00 \\
\hline & Rattus rattus & 00 & 00 & 00 & 00 & 01 & 00 & 01 & 0,63 \\
\hline & $\begin{array}{l}\text { Guerlinguetus } \\
\text { ingrami }\end{array}$ & $08(2)$ & $10(1)$ & 05 & $08(2)$ & 02 & $11(5)$ & $44(10)$ & $27,5(22,7)$ \\
\hline & $\begin{array}{l}\text { Sphigurus } \\
\text { villosus }\end{array}$ & 00 & 00 & 00 & 00 & 01 & 00 & 01 & 0,63 \\
\hline \multirow{6}{*}{ 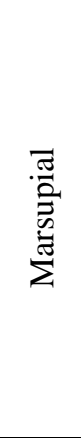 } & Didelphis aurita & $20(4)$ & $11(2)$ & 03 & $10(3)$ & 04 & $08(3)$ & $56(12)$ & $34,97(21,4)$ \\
\hline & $\begin{array}{l}\text { Marmosops } \\
\text { incanus }\end{array}$ & 04 & 05 & 03 & 05 & 00 & 00 & 17 & 10,63 \\
\hline & $\begin{array}{l}\text { Micoureus } \\
\text { paraguayanus }\end{array}$ & 04 & 02 & 00 & 09 & 01 & 02 & 18 & 11,25 \\
\hline & $\begin{array}{l}\text { Metachirus } \\
\text { nudicaudatus }\end{array}$ & 00 & 01 & 00 & 00 & 00 & 00 & 01 & 0,63 \\
\hline & $\begin{array}{l}\text { Monodelphis } \\
\text { americana }\end{array}$ & 00 & 00 & 01 & 00 & 00 & 02 & 03 & 1,88 \\
\hline & $\begin{array}{l}\text { Philander } \\
\text { frenatus }\end{array}$ & 00 & $01(1)$ & 00 & 00 & 00 & 00 & $01(1)$ & $0,63(100)$ \\
\hline \multirow[t]{2}{*}{ Total } & número & $38 \mathrm{a}$ & $36 a$ & $12 \mathrm{c}$ & $34 \mathrm{a}$ & $16 c$ & $24 \mathrm{~b}$ & $160(23)$ & $100(14,4)$ \\
\hline & porcentagem & 23,75 & 22,50 & 7,5 & 21,25 & 10,00 & 15,00 & 100 & \\
\hline \multicolumn{2}{|c|}{$\operatorname{IMP}\left(\mathrm{n}^{\mathrm{O}}\right)$} & $3,2 b$ & $12,0 \mathrm{a}$ & 0,0 & $2,8 \mathrm{~b}$ & 0,0 & $3,0 \mathrm{~b}$ & & 4,6 \\
\hline
\end{tabular}

$(\mathrm{p})$ = número de espécimes de hospedeiros parasitados por carrapatos entre os capturados na cota; (pi) = porcentagem de espécimes de hospedeiros parasitados por carrapatos entre os da mesma espécie. Expoentes com letras minúsculas iguais na mesma linha indicam diferença não significativa $(P>0,05)$, e com letras diferentes indicam diferença significativa $(\mathrm{P}<0,05)$.

O parasitismo de $I$. didelphidis, I. loricatus, I. auritulus e A. geayi em D. aurita é registrado pela primeira vez no município do Rio de Janeiro. A infestação simultânea de I. loricatus, I. amarali e A. geayi, em um mesmo hospedeiro (D. aurita), ainda não havia sido registrada em literatura. O parasitismo por formas adultas de $A$. longirostre em $G$. ingrami é achado incomum.

\section{CONCLUSÕES}

Houve correlação positiva e significativa entre a temperatura do ambiente e a infestação por carrapatos nos pequenos mamíferos do PEPB. Os roedores e marsupiais foram mais parasitados nas cotas mais baixas, que são áreas mais antropizadas. As espécies de ixodídeos mais frequentes foram Amblyomma longirostre, entre os roedores, e Ixodes loricatus, entre os marsupiais. 


\section{REFERÊNCIAS}

AMORIM, M.; SERRA-FREIRE, N.M. Descrição morfológica do estádio de larva de carrapato (Acari: Ixodidae). I. Amblyomma rotundatum Koch, 1844. Parasitol. al Día, v.19, p.9-19, 1995.

AMORIM, M.; SERRA-FREIRE N.M. Chave dicotômica para identificação de larvas de algumas espécies do gênero Amblyomma Koch, 1844 (Acari: Ixodidae). Entomol. y Vectores, v.6, p.75-90, 1999.

ARAGÃO, H.B. Ixodidas brasileiros e de alguns países limítrofes. Mem. Inst. Oswaldo Cruz, v.31, p.759-843, 1936.

ARAGÃO, H.B.; FONSECA, F. Notas de Ixodologia. VXXX. Lista e chave para os representantes da fauna ixodológica brasileira. Mem. Inst. Oswaldo Cruz, v.59, p.115-148, 1961.

ARZUA, M.; BARROS, D.M.; LINARDI, P.M Notework records of Ixodes auritulus Neumann, 1904 (Acari: Ixodida) on birds from Paraná, southern Brazil. Mem. Inst. Oswaldo Cruz, v.89, p.129, 1994.

BARROS-BATTESTI, D.M.; BAGGIO, D. Ectoparasites Ixodida Leach, 1817, on wild mammals in the state of Paraná, Brazil. Mem. Inst. Oswaldo Cruz, v.87, p.291-296, 1992.

BARROS-BATTESTI, D.M.; KNYSAK, I. Cataloge of the Brazilian Ixodes (Acari: Ixodidae) material in the Mite Collection of Instituto Butantan, São Paulo, Brazil. Papeis Avul. Zool., v.41, p.49-57, 1999.

BARROS-BATTESTI, D.M.; YOSHINARI, N.H.; BONOLD, V.L.N.; CASTRO, G.A. Parasitism by Ixodes didelphidis and Ixodes loricatus (Acari: Ixodidae) on Small Wild Mammals from Atlantic Forest in the State of São Paulo, Brazil. J. Med. Entomol., v.37, p.820-827, 2000.

BELDOMENICO, P.M.; LARESCHI, M.; NAVA, S. et al. The parasitism of immature stages of Ixodes loricatus (Acari: Ixodidae) on wild rodents in Argentina. Exp. Appl. Acarol., v.36, p.139-148, 2005.

BITTENCOURT, E.B.; ROCHA, C.F.D. Hostectoparasite specificity in a small mammals community in area of Atlantic Rain Forest (Ilha Grande, state of Rio de Janeiro), Southeastern Brazil. Mem. Inst. Oswaldo Cruz, v.98, p.793-798, 2003.

BOSSI, D.E.P.; LINHARES, A.X.; BERGALLO, H.G. Parasitic arthropods of some wild rodents from Juréia- Itatins Ecological Station, State of São Paulo, Brazil. Mem. Inst. Oswaldo Cruz, v.97, p.959-963, 2002.

BRUM, J.G.W.; VALENTE, A.L.S.; ALBANO, A.P. et al. Ixodidae de mamíferos silvestres atendidos no Núcleo de Reabilitação da Fauna Silvestre, UFPEL. Arq. Inst. Biol. São Paulo, v.70, p.211-212, 2003.
CINTRA, D.P.; OLIVEIRA, R.R.; REGO, L.F.G. Classificação de estágios sucessionais florestais através de imagens Ikonos no Parque Estadual da Pedra Branca, RJ. In: SIMPÓSIO BRASILEIRO DE SENSORIAMENTO REMOTO, 13. 2007, Santa Catarina. Anais... Florianópolis: [ s.n.] 2007. p.16271629.(Resumo)

COUTINHO, M.T.Z.; LINARDI, P.M.; BOTELHO, J.R. Ectoparasitos de Didelphis albiventris na Estação Ecológica da UFMG, Belo Horizonte, MG. In: CONGRESSO BRASILEIRO DE PARASITOLOGIA, 26., 1999, Minas Gerais. Anais... Poços de Caldas: [s.n.] 1999. p.59. (Resumo).

EVANS, D.E.; MARTINS, J.R.; GUGLIELMONE, A.A. A review of the ticks (Acari:Ixodidae) of Brazil, their hosts and geografic distribution - I The state of Rio Grande do Sul, southern Brazil. Mem. Inst. Oswaldo Cruz, v.95, p.453-470, 2000.

FONSECA, F. Inquérito sobre a fauna acarológica de parasitas no nordeste do Brasil. Mem. Inst. Butantan, v.28, p.99-186, 1957/58.

FONSECA, G.A.B. The vanishing Brazilian Atlantic Florest. Biol. Conservation, v.34, p.17-34, 1985.

FONSECA, F.; TRINDADE, G. Fauna acarológica de roedores em Ouro Preto. Mem. Inst. Oswaldo Cruz, v.28, p.59-66, 1957/58.

GONZALEZ-ACUNA, D.; VENZAL, J.M.; KEIRANS, J.E. et al. New host and locality records for the Ixodes auritulus (Acari: Ixodidae) species group, with a review of host relationship and distribution in the Neotropical Zoogeographic Region. Expl. Appl. Acarol., v.37, p.147-156, 2005.

GUGLIELMONE, A.A.; ESTRADA-PENA, A.; KEIRANS, J.E.; ROBBINS, R.G. Ticks (Acari: Ixodidae) of the Neotropical Zoogeographic Region. Netherlands: ICTTD-2, 2003a. 173p.

GUGLIELMONE, A.A.; ESTRADA-PENA, A.; MANGOLD, A.J. et al. Amblyomma aureolatum (Pallas, 1772) and Amblyomma ovale Koch, 1844 (Acari: Ixodidae): hosts, distribution and 16SrDNA sequences. Vet. Parasitol., v.113, p.273-288, 2003 b.

GUIMARÃES, J.H.; TUCCI, E.C.; BARROSBATTESTI, D.M. Ectoparasitos de importância veterinária. São Paulo: Pleiade, FAPESP, 2001. 218p.

GUITTON, N.; ARAÚJO FILHO, N.A.; SHERLOCK, I.A. Ectoparasitos de roedores e marsupiais no ambiente silvestre da Ilha Grande, estado do Rio de Janeiro, Brasil. Mem. Inst. Oswaldo Cruz, v.81, p.233-234, 1986. 
KEIRANS, J.E. Sistematics of the Ixodida (Argasidae, Ixodidae, Nutaliellidae): an overview and some problems. In: FIVAZ, B.; PETNEY, T.; HORAK, I. (Eds.). Tick vector biology medical and veterinary aspect. Berlin: Springer, 1992. p.1-21.

LINARDI, P.M.; BOTELHO, J.R.; NEVES, D.P.; CUNHA, H.C. Sobre alguns ectoparasitos de roedores silvestres de Belo Horizonte, Minas Gerais. Rev. Bras. Biol., v.44, p.215-219, 1984.

MULLER, G.; BRUM, J.G.W.; LANGONE, P.Q. et al. Didelphis albiventris Lund, 1841, parasitado por Ixodes loricatus Neumann, 1899, e Amblyomma aureolatum (Pallas, 1772) (Acari: Ixodidae) no Rio Grande do Sul. Arq. Inst. Biol. S. Paulo, v.72, p. 319324, 2005.
SERRA-FREIRE, N.M.; MELLO, R.P. Entomologia e Acarologia na Medicina Veterinária. Rio de Janeiro: L.F.Livros. 2006. 200p.

VENZAL, J.M.; CASTRO, O.; CLARAMUNT, S.; GUGLIELMONE, A.A. Primeiro registro de Amblyomma longirostre (Acari: Ixodidae) en Uruguay. Parasitol. Latinoam., v.58, p.72-74, 2003. 